\title{
木本植物木质部的冻融栓塞应对研究进展
}

\author{
李志民 王传宽
}

东北林业大学生态研究中心, 哈尔滨 150040 ; 森林生态系统可持续经营教育部重点实验室, 哈尔滨 150040

摘 要 冻融栓塞在中高纬度地区木本植物中普遍存在。抗冻融栓塞能力对在寒冷环境中木本植物的生长和安全越冬十分关 键, 这直接决定植物分布范围。冻融栓塞是由于冰中气体溶解度低, 木质部水分在低温下冷冻, 使之前水中溶解的气体逸出 到导管中, 随后木质部中的冰融化又使气泡扩张而引发的栓塞现象。木质部解剖结构的差异会影响植物的抗冻融栓塞能力, 植物还可以通过调节木质部正压、代谢耗能等方式主动修复冻融栓塞, 也可通过增加树液溶质含量等逃避冷冻, 以减少低温 损伤。然而, 与干旱栓塞相比, 目前对木质部冻融栓塞的形成以及植物响应和调节机制的理解不足。为此, 该文首先综述了 木质部冻融栓塞的形成机制和植物的逃避、忍耐、修复等 3 种冻融栓塞的应对策略, 然后总结了木质部抗低温胁迫能力的生 理表现、影响因子和评价指标, 并在此基础上讨论了低温抗性、干旱抗性和水力效率之间的多元权衡关系, 最后提出今后该 领域中的 5 个优先研究问题: (1)不同植物冰冻的最低温度阈值; (2)是否存在应对低温胁迫的水力脆弱性分割机制; (3)冻融栓 塞修复与代谢消耗的关系; (4)低温抗性、干旱抗性和水力效率之间的权衡关系; (5)抗冻融栓塞性状是否能够纳入经济性状谱 系。

关键词 冻融栓塞; 栓塞修复; 水力效率; 权衡; 水力特征

李志民, 王传宽 (2019). 木本植物木质部的冻融栓塞应对研究进展. 植物生态学报, 43, 635-647. DOI: 10.17521/cjpe.2019.0076

\section{Research progress on responses of xylem of woody plants to freeze-thaw embolism}

LI Zhi-Min and WANG Chuan-Kuan*

Center for Ecological Research, Northeast Forestry University, Harbin 150040, China; and Ministry of Education Key Laboratory of Sustainable Forest Ecosystem Management, Northeast Forestry University, Harbin 150040, China

\begin{abstract}
Embolism induced by the freeze-thaw commonly occurs in the woody plant in mid- and high-latitude regions. In cold environments, the resistance capacity to freeze-thaw embolism of woody plants is critical for their growth and successful survival throughout winter, which directly determines their distribution in the earth. During freeze-thaw cycles, the freeze-thaw embolism of vessels is induced by the bubbles that are from dissolved frozen water because of the low solubility of ice. The resistance capacity to freeze-thaw embolism varies from plants with different anatomical structures. Plants can also reduce potential damages of freeze-thaw embolism through adjusting the xylem positive pressure by refilling embolism and metabolism, and/or increasing the sap solute contents to avoid the stress. Compared to embolism induced by drought, however, few studies have been conducted on embolism by freeze-thaw, and the underlying mechanisms of plant responses and regulation are poorly understood. In this paper, we first reviewed the process of formation and repair of freeze-thaw embolism of xylem, including the strategies of escaping freezing, tolerance, and repairing freeze-thaw cavitation. Then we summarized physiological characteristics of plant resistance to low-temperature stress, influencing factors and evaluating criteria, based on the multiple trade-offs between low-temperature resistance, drought resistance, and hydraulic efficiency. Finally, we proposed five priorities for future study in this field: (1) the threshold of freezing temperature for different plants; (2) the vulnerability segmentation of frost-induced embolism; (3) the relationships between freeze-thaw cavitation repair and metabolism exhaustion; (4) the potential trade-offs between low-temperature resistance, drought resistance and hydraulic efficiency; and (5) integration of the traits of freeze-thaw embolism resistance into the economic spectrum of plants.
\end{abstract}

Key words freeze-thaw embolism; embolism repair; hydraulic efficiency; trade-off; hydraulic characteristic

收稿日期Received: 2019-04-07 接受日期Accepted: 2019-08-02

基金项目: 国家重点研发计划(2016YFD0600201)和教育部长江学者和创新团队发展计划(IRT_15R09)。Supported by the National Key R\&D Program of China (2016YFD0600201), and the Program for Changjiang Scholars and Innovative Research Team in University (IRT_15R09).

* 通信作者Corresponding author (wangck-cf@nefu.edu.cn) 
Li ZM, Wang CK (2019). Research progress on responses of xylem of woody plants to freeze-thaw embolism. Chinese Journal of Plant Ecology, 43, 635-647. DOI: 10.17521/cjpe.2019.0076

北方森林和温带森林几乎占全球森林总面积的 1/3 (Reich et al., 2016), 其植物面临的最主要环境 胁迫之一是冬季低温。低温使植物地上器官木质部 中的水分逐步冻结(Niu et al., 2017), 所以植物如何 最大程度减少低温胁迫引起的损伤对其安全越冬 (Hacke et al., 2015; Kreyling et al., 2015; Charrier et al., 2017)、生长、生存以及分布均具有重要作用 (Schreiber et al., 2013; Charrier et al., 2015; Niu et al., 2017)。低温时期冻融诱导形成的栓塞是低温胁迫对 植物产生损伤的重要因素(Zanne et al., 2014; Zhang et al., 2018)。许多研究发现, 植物抗冻融栓塞能力 不仅影响其在低温时期的损伤程度和碳消耗 (Bowling et al., 2018; Yin et al., 2018), 还显著影响 随后生长季中的水分供应能力(冷冻疲劳, Feng et al., 2015; Zhang et al., 2018)。但与干旱栓塞相比, 目 前对木质部冻融栓塞的形成以及植物响应和调节机 制的理解不足(Choat et al., 2011; Yin et al., 2018)。为 了推进该领域的研究, 本文先综述木质部冻融栓塞 的形成机制和植物的逃避、忍耐、修复等 3 种冻融栓 塞的应对策略, 然后总结植物低温抗性的生理表 现、评价和影响因子, 在此基础上讨论低温抗性、 干旱抗性和水力效率之间的多元权衡关系, 最后提 出今后该领域中的优先研究问题。

\section{1 木质部冻融栓塞的形成机理}

栓塞是降低木质部内水分运输效率的关键因子 (Choat et al., 2012; 金鹰等, 2016), 主要包括干旱栓 塞和冻融栓塞(Améglio et al., 2002; Willson \& Jackson, 2006; Venturas et al., 2017)。两种栓塞形成的主 要机理均为空气进入导管或管胞, 从而阻断木质部 水柱, 形成栓塞(Sperry \& Sullivan, 1992), 而不同之 处在于引发空气进入的过程 (Hacke \& Sperry, 2001)。根据内聚力-张力理论, 木质部导管内水分持 续运输动力主要来自叶片蒸腾失水引起的拉力, 因 此导管中的水分始终处于亚稳态 (Sperry \& Love, 2015)。当蒸腾加剧和/或土壤水分供应不足而使木 质部导管内负压超过一定阈值时, 就会造成其水柱 断裂, 致使周围组织中的气体进入导管, 从而形成 干旱栓塞(Choat et al., 2018)。而冻融栓塞主要发生 在木质部经历多次冻融循环的过程中(Sperry \&
Sullivan, 1992; Feild \& Brodribb, 2001; CharraVaskou et al., 2016)。当木质部水分冰冻时, 水中原 有溶解气体因在冰中的溶解度很低而逸散到导管中 (Lintunen et al., 2014)。而当冰冻融化时，导管中的 留存气体或者重新溶进水中(气泡压力大于大气压 时, Hacke \& Sperry, 2001), 或者由于冰冻压力的释 放(冰融化后体积减小) 等使气体在导管中扩大 (Lemoine et al., 1999; Mayr et al., 2002; Pittermann \& Sperry, 2006), 多次冻融交替之后, 导管中气体不 断增加和扩张, 最终引发冻融栓塞, 该现象也被称 为“融化-扩张假说” (Mayr \& Sperry, 2010; Mayr \& Améglio, 2016)。干旱栓塞和冻融栓塞均会阻碍植物 水分从土壤到叶片的运输, 从而降低叶片的光合速 率(Brodribb et al., 2007; Reich, 2014; Jin et al., 2016), 导致植物生产力降低甚至死亡(McDowell et al., 2008; Hoffmann et al., 2011; McDowell, 2011; Choat et al., 2018)。

\section{2 植物应对木质部冻融栓塞的策略}

在长期的进化过程中，植物(尤其是中高纬度、 高山和亚高山木本植物)面对冻融栓塞, 逐渐形成 了不同的应对策略(Lintunen et al., 2018; Wang et al., 2018; Willick et al., 2018; Yin et al., 2018), 主要包 括逃避、忍耐和修复等3种应对策略(图1)

\section{1 逃避策略}

冬季落叶是植物常见的逃避低温的策略之一 (Preston \& Sandve, 2013; Hoermiller et al., 2018)。乔 木和灌木树种通过调落叶片, 切断水力功能, 减少 低温胁迫损伤(Vitasse et al., 2014)。草本植物则通过 衰老地上组织, 以种子或者地下储存器官的形式, 在土壤和雪的保护下越冬(Zanne et al., 2014)。此外, 环孔材树种在低温时期直接使冻融栓塞的导管失去 生理功能, 而通过春季形成的新导管来运营其水分 运输功能, 这也是一种逃避低温策略(Cochard \& Tyree, 1990; Brodersen \& McElrone, 2013)。环孔材树 种的木质部水力效率较高, 其导管直径和长度也显 著大于散孔材树种，故其栓塞抗性较低(殷笑寒和 郝广友, 2018)。

植物还可以通过木质部过冷却的方式避免低温 及冰冻损伤(Arias et al., 2017)。过冷却指植物使细 


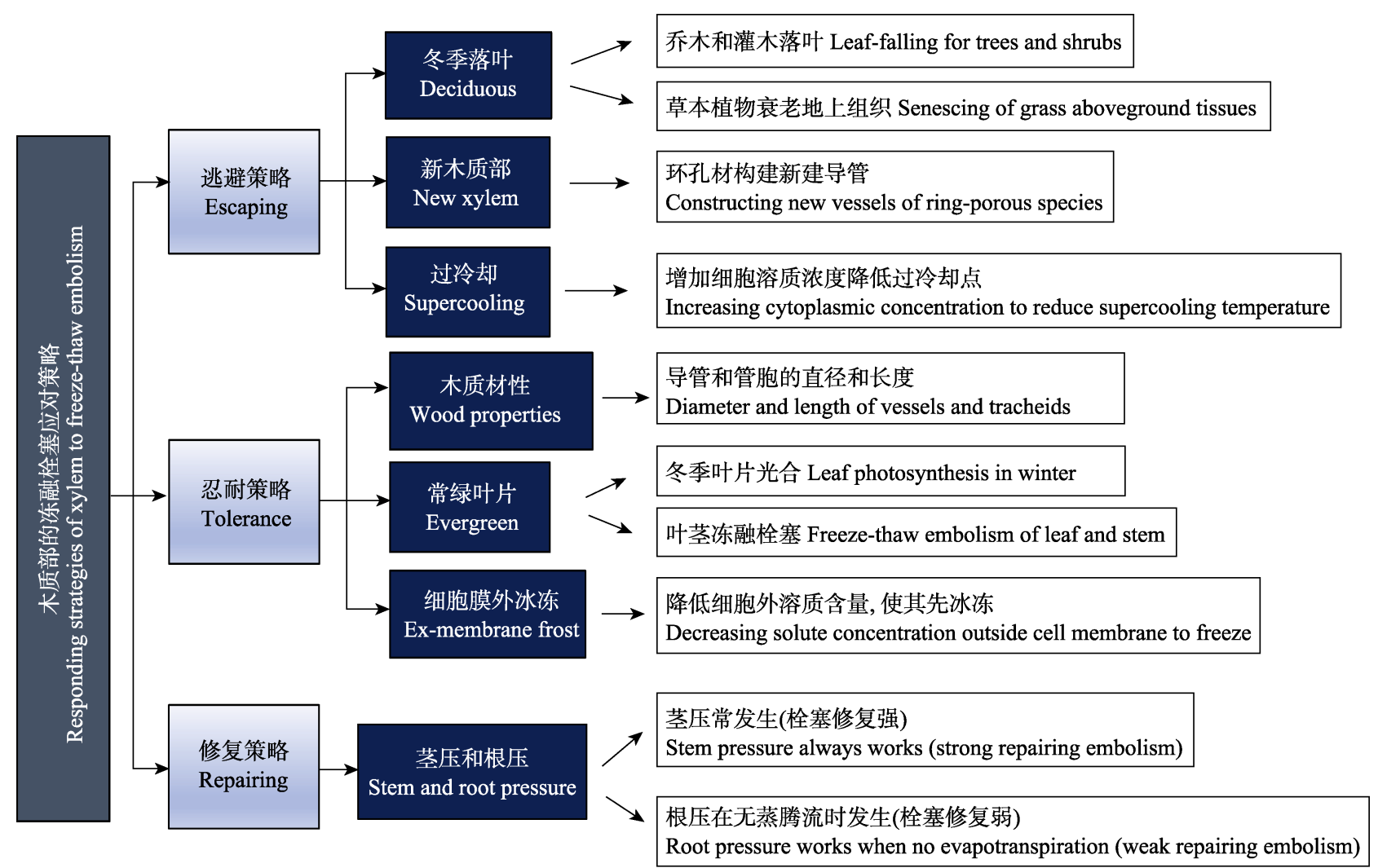

图1 植物木质部的冻融栓塞应对策略。

Fig. 1 Three responding strategies of xylem to freeze-thaw embolism.

胞中液体的冰点温度远低于液态水冰点的能力 (Arias et al., 2015)。Rasmussen和MacKenzie (1972) 指出, 没有任何结晶核的纯水结冰温度低至 $-38.1{ }^{\circ} \mathrm{C}$ 。因此, 植物细胞内异质结晶核含量影响其 过冷却点的高低(Losso et al., 2018)。过冷却能力对 植物应对突发性的冰冻事件(如热带植物遭遇突发 性寒流)十分重要(Reyes-Díaz et al., 2006)。Arias等 (2015)发现, 在寒冷环境中驯化过的油橄榄(Olea europaea)栽培种, 其叶片冰点温度低至 $-13{ }^{\circ} \mathrm{C}$ (远 低于环境最低温度), 而且有更高的叶密度和细胞 壁刚性, 从而使其溶质渗透性和组织可塑性更高, 质外体水分含量更低; 进一步研究发现, 从夏季到 冬季该树种茎最低冰点逐渐降低, 而且其木质部抗 空穴化能力与叶片和茎的过冷却能力之间存在权衡 (Arias et al., 2017)。

\section{2 忍耐策略}

与动物不同, 植物的固定性使得其更多地采用 “被动”的忍耐策略, 例如不同的木质结构、调整组 织水分含量和溶质含量等, 以减少低温损伤(Mayr et al., 2014; Arias et al., 2015; Lintunen et al., 2018)。 被子植物和裸子植物解剖结构的差异, 使二者对低
温胁迫的忍耐能力不同(Pittermann \& Sperry, 2003; Lintunen et al., 2016)。被子植物的导管比裸子植物 的管胞结构具有更高的水力效率, 但是其木质部抗 冻融栓塞能力较低(Feild \& Brodribb, 2001; Lintunen et al., 2018)。Davis等(1999)报道, 当导管直径大于 $40 \mu \mathrm{m}$ 时, 一次冻融交替使木质部栓塞程度高达 95\%; Feild和Brodribb (2001)也发现, 常绿针叶植物 和导管较小的被子植物经历一次冻融交替后, 其茎 水力导度降低最少 (几乎为 0 ), 而其他被子植物则平 均降低 $18 \%-70 \%$ 。可见, 被子植物木质部忍耐低温 能力远低于裸子植物。植物经过多次冻融交替后, 导管或周围组织中产生的气泡可能在整个冬季留存, 直到春季才能重新进入融化水中或者气泡扩张形成 栓塞(Hacke \& Sperry, 2001; Venturas et al., 2017; Medeiros \& Danielson, 2018), 所以导管内水分含量 直接影响植物冻融栓塞程度(Choat et al., 2011)。而 水分含量与导管直径紧密相关(Lens et al., 2013)。因 此构建直径更小的导管是最直接增加抗冻融栓塞能 力的方法。裸子植物直径较小的管胞结构能够在很 大程度上减少冻融栓塞的形成, 不仅因为在管胞中 形成的气泡更小, 而且小气泡更容易在融化期重新 
溶解进入水中(Hacke et al., 2015)。Pittermann和 Sperry等(2006)报道，管胞越窄的裸子树种气泡形 成的空穴化越小, 并且得出冻融栓塞引起的木质部 负压随着管胞直径的增加而增加。之后, Choat等 (2011)对11种被子植物的研究也发现, 导管直径越 大、长度越长的植物, 冻融引起的水力导度丢失量 (PLC) 越多、造成的栓塞越大。这可能是因为直径较 大的导管具有更少的单位长度导管末端, 造成其纹 孔数量减少、栓塞抗性降低(Choat et al., 2006)。因 此, 构建直径更小的导管(或管胞), 是植物应对冻 融栓塞最直接的方式(Pittermann \& Sperry, 2006; Jiménez-Castillo \& Lusk, 2013; Hacke et al., 2017; Gleason et al., 2018)。

常绿树种茎抗低温胁迫能力可能还与叶片低温 抗性有关(Ball et al., 2004; Fernández-Pérez et al., 2018; Verhoeven et al., 2018)。“脆弱性分割假说”指 出, 植物面临胁迫时, 其叶片(或小枝)可能比其茎 表现得更为脆弱, 通过牺牲 “造价低廉”的末端器官 保护“造价昂贵”的茎干, 以避免栓塞发生(Zimmermann, 1983; Tyree \& Ewers, 1991)。已存在大量有关 干旱胁迫下的“脆弱性分割假说”验证研究(Hao et al., 2008; Johnson et al., 2012; Pivovaroff et al., 2014; Zhu et al., 2019), 但还不清楚低温胁迫下是否也会 发生该现象。Fernández-Pérez等(2018)发现, 6 个松属 (Pinus) 树种的针叶低温抗性对其分布范围的预测能 力大于茎干木质部抗冻融栓塞能力。在北半球温带 森林中, 冬季留存叶片因日间光照而使其日间温度 高于 $0{ }^{\circ} \mathrm{C}$, 从而可能延长光合作用的时间(Feild \& Brodribb, 2001; Sevanto et al., 2006; Lindfors et al., 2015; Bowling et al., 2018), 生产的有机物可用于早 春修复冻融栓塞和细胞损伤。不过, 此时树干仍处 于冰冻状态, 导致叶片可能比茎经历更多次的冻融 循环, 从而加剧叶片的冻融栓塞(Feild \& Brodribb, 2001; Charra-Vaskou et al., 2016), 降低茎干的冻融 栓塞程度, 在一定程度上对主茎起到保护作用 (Charrier et al., 2017)。

植物还有一种忍耐低温胁迫的策略是, 通过改 变细胞膜内外的溶质浓度, 使细胞内溶质浓度增加 (形成低水势), 从而降低细胞外溶质浓度(Mayr et al., 2007; Lintunen et al., 2016)。当冰冻发生时, 细胞 外水分首先冻结(主要发生在细胞壁表面、纤维、导
管内或者细胞外的空间)(Willick et al., 2018), 冰形 成的低水势使细胞内水分进一步流出, 细胞内溶质 浓度增加, 从而避免细胞内冰冻导致的细胞死亡 (McCully et al., 2004), 能够暂时保护细胞不受冰冻 损伤(Mayr \& Améglio, 2016)。

\section{3 修复策略}

修复是植物应对冻融栓塞的又一重要策略 (Preston \& Sandve, 2013; Wang et al., 2018)。有研究 表明, 采用正压(主要包括根压和茎压)恢复水力功 能的植物可能具有更强的抗冷冻疲劳能力(即木质 部冰冻后对水力功能丢失更敏感的现象)(Niu et al., 2017; Yin et al., 2018), 这是植物对冻融栓塞的一种 主动修复。Hao等(2013)发现, 早春纸桦(Betula papyrifera)树干水分含量明显高于生长季, 表明根压 在修复冻融栓塞导管的过程中具有重要作用。 Améglio等(2002)报道, 冬季胡桃(Juglans regia) $P L C$ 可高达到 $100 \%$, 但是由于冬季和春季茎压修复, 木质部冻融栓塞程度有一定的降低。另外, 近期研 究表明, 能产生木质部正压的植物, 其抗冻融栓塞 能力可能不受导管直径的影响(Niu et al., 2017)。根 压和茎压主要来源于糖类水解成的蔗糖被运输到栓 塞导管中而产生渗透浓度梯度(Nardini et al., 2011; Cao et al., 2012), 从而使水分流向栓塞导管, 即渗 透调节假说(金鹰等, 2016)。两者的不同之处在于: 根压主要在无蒸腾流情况下产生作用(Swaef et al., 2013), 且修复栓塞作用较小(Salleo et al., 2004), 特 别是对高大植物上层木质部栓塞的修复作用很小 (Westhoff et al., 2008)。而茎压产生的渗透梯度可能 来自韧皮部的糖类(韧皮部卸载假说, Salleo et al., 2004; Hölttä et al., 2018), 因此在多数情况下均有茎 压存在，这也被称为“新的再冲水” (novel refilling) 假说(Brodersen \& McElrone, 2013; 金鹰等, 2016)。 Améglio等(2004)发现, 胡桃在深冬时茎中淀粉含量 最低, 在发芽前茎中再次合成淀粉, 这不仅表明胡 桃在冬季低温下进行淀粉和蔗糖的转换, 而且蔗糖 累积是其冻融栓塞修复中的关键。张海燕等(2013) 也发现, 12 个温带树种老枝的淀粉含量变化具有相 同趋势。

\section{3 植物木质部低温胁迫抗性和影响因子}

木质部结构是影响植物忍耐低温胁迫的主要因 
素(Hacke et al., 2015, 2017; Lintunen et al., 2018)。被 子植物采用多个细胞死亡后相互连接构成的导管进 行水分运输, 长度在 $1 \mathrm{~mm}$ 到几米不等; 裸子植物则 依靠单个细胞伸长形成的管胞进行水分运输, 长度 在2-6 mm之间(Tyree \& Ewers, 1991), 而且管胞直 径一般小于导管(Sperry et al., 2006)。这种木质部结 构差异导致裸子植物和被子植物响应冻融栓塞时产 生显著PLC差异(Feild \& Brodribb, 2001; Feng et al., 2015; Fernández-Pérez et al., 2018)。以往研究表明, 植物在入冬前PLC逐步增加(Zhang et al., 2018), 而 导管或管胞的直径差异是植物 $P L C$ 不同的主要原因 (Pittermann \& Sperry, 2003; Hacke et al., 2015, 2017)。在所有季节中, 裸子植物的PLC均小于被子 植物(Granda et al., 2014)。例如, 被子植物葡萄牙栎 (Quercus faginea)抗空穴化能力较差, 夏季PLC达到 $80 \%$, 冬季进一步达到 $90 \%$, 而裸子植物香桧 (Juniperus thurifera)和欧洲黑松(Pinus nigra)夏季 $P L C$ 几乎为 0 , 冬季仅增加到 $3 \%-9 \%$ (Granda et al., 2014)。

经典气泡扩张理论认为, 冻融栓塞的形成取决 于融化时木质部张力 $\left(P_{\mathrm{x}}\right)$ 和气泡压力 $\left(P^{*}\right)$ 之间的关 系(Domec, 2011; Charrier et al., 2017); $P^{*}$ 受气泡表 面张力 $(t)$ 和气泡中气液混合物压力 $\left(P_{\mathrm{b}}\right)$ 的共同影响, $P^{*}$ 还与气泡直径 $\left(D_{\mathrm{b}}\right)$ 大小相关。因此,

$$
P^{*}=-4 t / D_{\mathrm{b}}+P_{\mathrm{b}}
$$

只有当 $P_{\mathrm{x}}$ 小于 $P^{*}$ 时, 气泡才有可能扩大并形成 空穴。由公式(1)可知, 木质部导管越大, 越容易形 成直径较大的气泡, 气泡压力越小, 在较低张力下 气泡越可能扩大, 因而对冻融栓塞的抵抗更加脆弱 (Feild \& Brodribb, 2001; Choat et al., 2011; Hacke et al., 2017)。裸子植物管胞直径一般比被子植物导管 直径小(Hacke et al., 2017), 因此裸子植物的抗冻融 栓塞能力较强。但在管道直径相同时, 裸子植物与 被子植物具有相似的抗冻融栓塞能力 (Pittermann \& Sperry, 2003, 2006; Hacke et al., 2015)。

木质部水分含量是影响植物抗冻融栓塞能力的 又一因素。因为导管直径与木质部水分含量正相关 (Lens et al., 2013), 因此, 裸子植物管胞的含水量一 般低于被子植物导管, 从而使裸子植物的冻融栓塞 程度较低。冬季日间太阳直射使留存的植物叶片和/ 或小枝中冰冻水分融化, 引起蒸腾失水, 但下部冰
冻的树干(Charrier et al., 2017)无法及时补充水分丢 失(Bowling et al., 2018), 从而使植物叶片和小枝的 木质部含水量降低。另外, 含水量变化还可能会引 起质外体组织冰冻时的体积发生变化(Lintunen et al., 2016)。低温胁迫使木质组织首先脱水收缩, 再 经历液态水冰冻时的体积膨大 $(+9 \%)$ (Améglio et al., 2001)。在冰冻前, 植物主动降低质外体含水量, 增 加溶质浓度, 此时脱水引起的体积减小能够降低过 冷却点(Lintunen et al., 2013; Willick et al., 2019)。因 此, 冰冻前含水量越低(体积越小), 植物对低温胁 迫的忍耐力越强(Lintunen et al., 2018)。当冰冻发生 时, 由于水分由液态转为固态的体积增加, 对周围 细胞壁产生机械压力, 植物需构建更强健的细胞壁 (Zhang et al., 2016; Ployet et al., 2018)和改变细胞壁 上蛋白组分(Willick et al., 2018)来抵御低温胁迫, 因此, 质外体较高的水分含量可能对植物产生更严 重的低温损伤。研究表明, 木质部通过寒冷驯化 后其直径少量收缩(Améglio et al., 2001; CharraVaskou et al., 2016; Lintunen et al., 2016)。例如，欧 洲赤松(Pinus sylvestris)和欧洲山杨(Populus tremula) 木质部直径经寒冷驯化后分别收缩了 $0.5 \%$ 和 $1.0 \%$, 小于渗透胁迫和干旱胁迫引起的收缩量(Lintunen et $a l ., 2016)$ 。此外, 植物通过寒冷驯化, 可以降低质外 体的含水量, 并将水分转移到冰储存位点, 同时不 同冰区之间形成隔离带(Willick et al., 2019), 从而 尽可能减少低温损伤。

\section{4 评价植物抗低温胁迫能力的生理指标}

评价植物抗冻融栓塞能力最直接的指标是冻融 栓塞引起水力导度丢失的百分比, 即木质部最大水 力导度与低温下水力导度之差和最大水力导度之比 (Charrier et al., 2013; Niu et al., 2017), 与干旱PLC 类似。冻融栓塞常常会引起植物水力导度丢失现象。 Granda等(2014)整合75个研究中的 210 个记录发现, 植物PLC从夏季到冬季逐步增加。Charrier等(2013) 也发现, 冬季植物 $P L C$ 最高, 且 $P L C$ 可能受海拔限 制。然而, Fernández-Pérez等(2018)报道, 低温胁迫 引起的 6 个松属树种的 $P L C$ 较低, 而且没有发现PLC 与低温损伤(叶黎明前水势)之间的关系。

虽然植物通过逃避、忍耐、修复等方式应对冻 融栓塞, 但冻融过程中导管细胞壁和纹孔膜的损伤 会显著降低植物再次应对冻融栓塞的能力, 该现象 
被称为“冷冻疲劳” (Christensen-Dalsgaard \& Tyree, 2013)。此概念最初源于木质部在经历多次干旱栓塞 后, 再次面临干旱时会增加栓塞敏感性, 降低植物 抗干旱栓塞能力(Stiller \& Sperry, 2002; Hillabrand et al., 2016)。同样, 冷冻疲劳指木质部经过多次冻融 交替后对水力丢失更为敏感(Yin et al., 2018)。通常 采用自然条件下导水率丢失 $50 \%$ 时所对应的水势 $\left(P_{50}\right)$ 与经过一次(或多次)冻融交替后的 $P_{50}$ 之差表示 冷冻疲劳(Christensen-Dalsgaard \& Tyree, 2014; Feng et al., 2015; Zhang et al., 2018)。ChristensenDalsgaard和Tyree (2014)发现, 经过一个自然条件下 冬季后, 散孔材树种的栓塞抗性显著降低, 导水率 丢失 $25 \%$ 时所对应的水势 $\left(P_{25}\right)$ 比 $P_{50}$ 降低更多, 这表 明散孔材树种表现出显著的冷冻疲劳。Yin等(2018) 发现, 在模拟冻融交替下, 不同功能型树种的冷冻 疲劳(根压、根压+茎压、无根压、茎压)差异显著, 且 与木质密度、导管密度和单个纹孔特性有关。与茎 压相比, 能够产生根压和根压+茎压的树种所具有 的更高木质密度, 可以增加其忍耐木质部被拉长 (冰冻体积增加)的能力(Yin et al., 2018)。Zhang等 (2018)报道, 五角槭(Acer mono)冷冻疲劳具有循环 周期性, 表现出冬季最弱、夏季最高。上述研究结 果表明, 冷冻疲劳可能是温带森林中普遍存在的现 象(Christensen-Dalsgaard \& Tyree, 2013), 并且显著 影响植物抗冻融栓塞能力。

另一种评价植物低温耐性的生理特征是低温对 植物的损伤程度, 即将经过低温处理后的植物置于 适宜环境中生长, 之后观察枯落叶(或者死根)的百 分比, 以此来判断植物低温忍耐能力(Carles et al., 2011; Rorato et al., 2018)。Toca等(2018)报道, 经历 冬季(低温处理)后的松树幼苗, 其小枝低温忍耐能 力明显增加, 且氮添加显著影响植物低温忍耐能力, 但正负效应均存在。土壤养分是否影响植物低温耐 性还有待于深入研究。

常绿植物保证叶片安全越冬对其之后的生长具 有关键作用(Ball et al., 2004), 因此我们还可以通过 分析叶片抗低温胁迫能力来评价植物的低温耐性。 目前评价叶片抗低温胁迫能力的指标主要有: 叶片 电解质渗出率(Climent et al., 2009; Hoermiller et al., 2018)、可溶性糖含量(Fernández-Pérez et al., 2018)、 黎明前叶水势(Taneda \& Tateno, 2005)、叶细胞弹性
模量(细胞壁刚性越高, 细胞弹性模量越大)(Zhang et al., 2016)等。Fernández-Pérez等(2018)报道，伊比 利亚半岛 6 个松属树种的分布与其叶片低温忍耐能 力有关, 生长在寒冷环境中的针叶的电解质渗出率 和可溶性糖含量较低, 且黎明前叶水势对针叶低温 损伤有影响。Martínez-Vilalta等(2016)整合分析全球 尺度 121 个研究中 177 个物种非结构性碳(淀粉和可 溶性糖)数据发现，北方和温带生物区系物种叶片 非结构性碳含量最高, 而且在入冬过程中逐步增 加。Zhang等(2016)发现，叶片细胞壁强度的增加， 降低了质外体冰冻对细胞膜的物理损伤, 使植物在 较低的温度下得以存活。上述几个评价指标中, 电 解质渗出率是最常用的评价植物组织损伤的指标 (Charrier et al., 2013; Arias et al., 2015; Mayr \& Améglio, 2016)。通常采用电解质渗出率达 $50 \%$ 时所 对应的温度 $\left(L T_{50}\right)$ 来评价植物的低温抗性: $L T_{50}$ 越低, 则低温抗性越强(Kreyling et al., 2015; Bachofen et al., 2016; Arias et al., 2017)。例如, Arias等(2017)报 道, 油橄榄的 $L T_{50}$ 从夏季到冬季呈现逐步降低的趋 势, 这表明随着温度的降低, 通过不断驯化, 油橄 榄的低温抗性逐步增强。

\section{5 木质部低温抗性、干旱抗性和水力效率的 权衡}

木质部的水力效率-安全权衡是植物适应环境 和进化的结果(Blackman et al., 2010; Jin et al., 2016), 影响植物的竞争力和生产力 (Nardini \& Luglio, 2014)。水力效率一般用植物水力导度表示, 而水力 安全常用抗干旱栓塞能力 $\left(P_{50}\right)$ 表示(Santiago et al., 2018)。Nardini等(2012)报道，来自温带的6种植物 (槭属(Acer)和栎属(Quercus)各3种)的水力导度、 $P_{50}$ 和比叶质量之间存在显著相关性, 支持水力效率安全权衡, 且增加叶碳投入可以提高水力丢失的忍 耐能力。Nardini和Luglio (2014)整合130个木本被子 植物叶片水力数据后也发现存在叶水力效率-安全 权衡关系, 而且生长在低降水量地区的植物的 $P_{50}$ (负值)和叶水力导度均较低。Gleason等(2016)综合 分析 335 种被子植物和 89 种裸子植物的茎水力数据, 发现虽然水力导度和抗干旱栓塞能力之间存在弱相 关性(支持水力效率-安全权衡), 但没有发现高效率 和高安全性的植物。因此, 植物普遍存在不同程度 
的水力效率-安全权衡关系, 但其关系的紧密程度 受导管特征(Lens et al., 2011; Hajek et al., 2014)、木 质材性(金鹰和王传宽，2016)、树高(Prendin et al., 2018)等因素的影响。

与干旱栓塞类似, 冻融栓塞也阻断木质部的水 分运输, 由此推测水力导度随着抗冻融栓塞能力的 增加而降低, 然而, 以往研究结果并不一致。例如, Choat等(2011)研究发现, 温带树种水分运输能力与 冻融栓塞之间存在权衡, 且该权衡影响光合作用。 但Feild和Brodribb (2001)报道, 低温PLC与茎水力 导度无关, 不支持抗冻融栓塞能力与水力导度之间 的权衡。Niu等(2017)也发现, 散孔材树种能产生木 质部正压, 不存在水力效率和抗冻融栓塞能力的权 衡现象, 而其他功能型树种则存在这种权衡关系, 这表明根压或茎压会影响该权衡。因此，抗冻融栓 塞能力与水力导度之间的权衡是否普遍存在, 尚需 进一步研究。

木质部低温抗性、干旱抗性和水力效率三者间 是否存在多元权衡现象? 目前对此问题的综合研究 较少, 但已有研究指出, 干旱显著增加植物的低温 抗性(Kreyling et al., 2012; Walter et al., 2013; SierraAlmeida et al., 2016; Sperling et al., 2017)。这是因为 植物通过驯化改变了细胞对脱水的忍耐能力 (Buchner \& Neuner, 2011), 其中包括基因表达的改 变导致的细胞膜结构变化或者细胞化学成分变化 (Verhoeven et al., 2018)。例如, Medeiros和Pockman (2011)报道, 在-15 ${ }^{\circ} \mathrm{C}$ 环境中干旱处理使Larrea tridentate植株细胞死亡减少、叶留存量增加、水力导 度和气体交换速率升高, 这表明干旱处理显著增加 了其低温抗性。Hoffman等(2012)也发现, 干旱处理 能够显著增加黑麦草(Lolium perenne)的低温抗性。 然而, Kong和Henry (2016)研究发现, 早春低温处理 的草地早熟禾(Poa pratensis)的抗旱性显著增加(生 物量和存活率增加), 但夏季干旱处理并未增加其 低温抗性, 这表明干旱处理对植物低温抗性无影响 的现象也存在。同样, Bachofen等(2016)也发现, 干 旱处理没有增加地中海松属 3 个树种的低温抗性, 而且推测该现象可能仅在短期极端条件下发生。Yin 等(2018)同时对低温抗性、干旱抗性和水力效率进 行研究发现, 抗干旱栓塞与水力效率存在权衡, 抗 冷冻疲劳与水力效率间也存在权衡现象, 支持多元
权衡现象。然而，当植物同时面对干旱和低温胁迫 时(如高山树线植物)(Mayr et al., 2007; Charrier et al., 2017), 多次冻融交替结合干旱胁迫可能引起 PLC增加(Mayr et al., 2003; Charrier et al., 2014)。 Mayr等(2006)报道, 生长在林线处的针叶树种, 每 个冬季经历至少 100 次冻融交替, 再加上干旱胁迫, 二者共同引起欧洲云杉(Picea abies) PLC高达 $100 \%$ 。因此, 生长在低温和干旱胁迫共存环境中的 植物, 其 $P L C$ 可能更高, 间接表明三者间存在一定 程度的权衡。综上所述, 我们构建了低温抗性、干 旱抗性和水力效率之间多元权衡概念图(图2)。

\section{6 研究问题和展望}

许多研究表明，植物木质部在环境温度低于 $0{ }^{\circ} \mathrm{C}$ 后没有立即发生冰冻现象。植物应对低温的忍 耐策略(过冷却能力和降低水势等)能够降低形成冰 冻的最低温度。因此, 明确不同植物冰冻的最低温 度阈值能够提高在全球气候变化条件下对植物分布 的预测能力。

植物不同器官对干旱胁迫的响应存在差异, 叶 片(或小枝)比主茎抗干旱栓塞能力更弱，即“脆弱性 分割假说”。冻融栓塞和干旱栓塞均能阻碍水分运 输。因此, 植物不同器官间的抗冻融栓塞能力是否 也存在分割现象, 这如何影响植物抗低温胁迫能力 等相关问题仍需进一步研究。

植物主动修复冻融栓塞的过程是将溶质主动运 输到栓塞导管中以形成渗透势梯度，这会产生碳消 耗，因此，该过程可能与 $\mathrm{CO}_{2}$ 释放有关。随着全球温 度升高, 低温胁迫对植物的损伤可能减轻, 从而降 低植物用于修复冻融栓塞的代谢消耗，这可能会影 响植物的碳固定量, 但需要更多的实验验证。

探索木质部低温抗性、干旱抗性和水力效率间 的多元权衡, 深入了解干旱和低温胁迫对植物生理 特征的影响, 特别是植物如何调整生理结构和代谢 来适应干旱和低温胁迫共存的环境。这对植物应对 不断加剧的全球气候变化的研究具有重要意义。

Reich (2014)提出植物体中存在“快-慢”谱系的 普适性规律，包括碳、氮和水分性状的耦合关系，但 没有明确包含植物低温时期的生理特征。植物抗低 温胁迫能力是否能够纳入经济性状谱系，与其他经 济性状是否相关等问题亟待解决。 


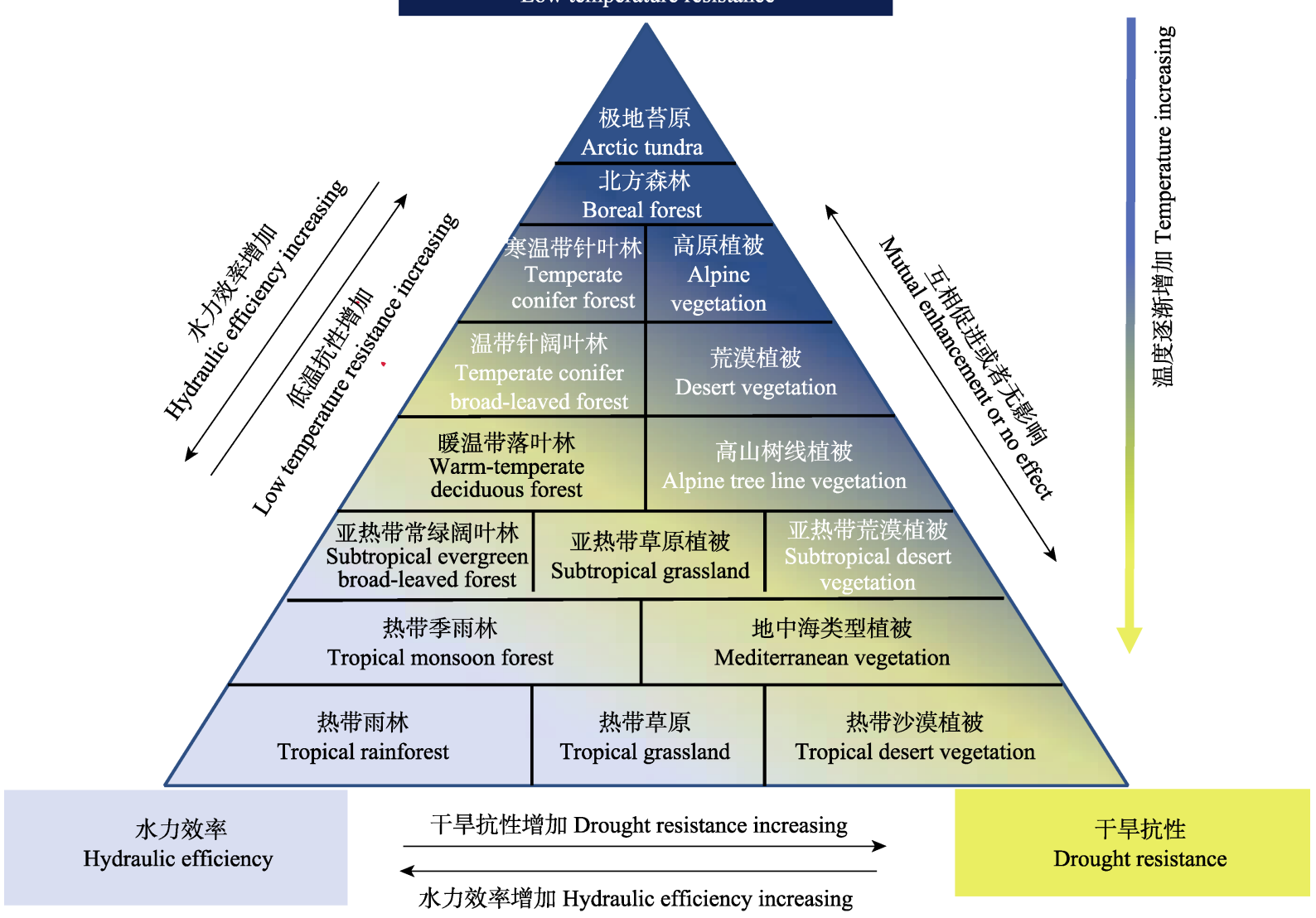

水分逐渐增加 Water availability increasing

图2 主要植被类型低温抗性、干旱抗性和水力效率之间多元权衡的概念图。高(低)水力效率和低(高)低温抗性相关联, 但主 动修复可影响该权衡，低温还可影响植物的冷冻瘦劳; 高(低)水力效率与低(高)干旱抗性相关联，几乎不存在同时高水力效率 和高干旱抗性的植物，但具有低水力效率和低干旱抗性的植物; 干旱胁迫可增加低温抗性(不同时), 反之亦然，干旱和低温同 时发生增加水力导度丢失量, 但两者对水力系统的影响并不相关。

Fig. 2 A conceptual diagram of multiple trade-offs among low-temperature resistance, drought resistance and hydraulic efficiency of the major vegetation types in the world. High (low) hydraulic efficiency is associated with weak (strong) low-temperature resistance, but the capacity of positive refilling embolism may influence the trade-off between hydraulic efficiency and low-temperature resistance, and low-temperature also affects the plant's frost fatigue. High (low) hydraulic efficiency is associated with weak (strong) drought resistance, but many species have both low hydraulic efficiency and drought resistance rather than high hydraulic efficiency and drought resistance. Drought stress may increase low-temperature resistance (asynchronous), and vice versa; concomitant drought and low-temperature stresses aggravate the loss of hydraulic conductance, but there are no related impacts of drought and low-temperature stresses on the hydraulic system.

\section{参考文献}

Améglio T, Bodet C, Lacointe A, Cochard H (2002). Winter embolism, mechanisms of xylem hydraulic conductivity recovery and springtime growth patterns in walnut and peach trees. Tree Physiology, 22, 1211-1220.

Améglio T, Cochard H, Ewers FW (2001). Stem diameter variations and cold hardiness in walnut trees. Journal of Experimental Botany, 52, 2135-2142.

Améglio T, Decourteix M, Alves G, Valentin V, Sakr S, Julien JL, Petel G, Guilliot A, Lacointe A (2004). Temperature effects on xylem sap osmolarity in walnut trees: Evidence for a vitalistic model of winter embolism repair. Tree
Physiology, 24, 785-793.

Arias NS, Bucci SJ, Scholz FG, Goldstein G (2015). Freezing avoidance by supercooling in Olea europaea cultivars: The role of apoplastic water, solute content and cell wall rigidity. Plant, Cell \& Environment, 38, 2061-2070.

Arias NS, Scholz FG, Goldstein G, Bucci SJ (2017). The cost of avoiding freezing in stems: Trade-off between xylem resistance to cavitation and supercooling capacity in woody plants. Tree Physiology, 37, 1251-1262.

Bachofen C, Wohlgemuth T, Ghazoul J, Moser B (2016). Cold temperature extremes during spring do not limit the range shift of Mediterranean pines into regions with intermittent frost. Functional Ecology, 30, 856-865.

www.plant-ecology.com 
Ball MC, Canny MJ, Huang CX, Heady RD (2004). Structural changes in acclimated and unacclimated leaves during freezing and thawing. Functional Plant Biology, 31, 29-40.

Blackman CJ, Brodribb TJ, Jordan GJ (2010). Leaf hydraulic vulnerability is related to conduit dimensions and drought resistance across a diverse range of woody angiosperms. New Phytologist, 188, 1113-1123.

Bowling DR, Logan BA, Hufkens K, Aubrecht DM, Richardson AD, Burns SP, Anderegg WRL, Blanken PD, Eiriksson DP (2018). Limitations to winter and spring photosynthesis of a Rocky Mountain subalpine forest. Agricultural and Forest Meteorology, 252, 241-255.

Brodersen CR, McElrone AJ (2013). Maintenance of xylem network transport capacity: A review of embolism repair in vascular plants. Frontiers in Plant Science, 4, 108. DOI: 10.3389/fpls.2013.00108.

Brodribb TJ, Feild TS, Jordan GJ (2007). Leaf maximum photosynthetic rate and venation are linked by hydraulics. Plant Physiology, 144, 1890-1898.

Buchner O, Neuner G (2011). Winter frost resistance of Pinus cembra measured in situ at the alpine timberline as affected by temperature conditions. Tree Physiology, 31, 1217-1227.

Cao KF, Yang SJ, Zhang YJ, Brodribb TJ (2012). The maximum height of grasses is determined by roots. Ecology Letters, 15, 666-672.

Carles S, Lamhamedi MS, Stowe DC, Bernier PY, Veilleux L, Margolis HA (2011). Relationships between frost hardiness, root growth potential, and photosynthesis of nurserygrown white spruce seedlings. Annals of Forest Science, 68, 1303-1313.

Charra-Vaskou K, Badel E, Charrier G, Ponomarenko A, Bonhomme M, Foucat L, Mayr S, Améglio T (2016). Cavitation and water fluxes driven by ice water potential in Juglans regia during freeze-thaw cycles. Journal of Experimental Botany, 67, 739-750.

Charrier G, Charra-Vaskou K, Kasuga J, Cochard H, Mayr S, Améglio T (2014). Freeze-thaw stress: Effects of temperature on hydraulic conductivity and ultrasonic activity in ten woody angiosperms. Plant Physiology, 164, 992-998.

Charrier G, Cochard H, Améglio T (2013). Evaluation of the impact of frost resistances on potential altitudinal limit of trees. Tree Physiology, 33, 891-902.

Charrier G, Ngao J, Saudreau M, Améglio T (2015). Effects of environmental factors and management practices on microclimate, winter physiology, and frost resistance in trees. Frontiers in Plant Science, 6, 259. DOI: 10.3389/fpls.2015. 00259 .

Charrier G, Nolf M, Leitinger G, Charra-Vaskou K, Losso A, Tappeiner U, Améglio T, Mayr S (2017). Monitoring of freezing dynamics in trees: A simple phase shift causes complexity. Plant Physiology, 173, 2196-2207.

Choat B, Brodie TW, Cobb AR, Zwieniecki MA, Holbrook NM (2006). Direct measurements of intervessel pit membrane hydraulic resistance in two angiosperm tree species. American Journal of Botany, 93, 993-1000.

Choat B, Brodribb TJ, Brodersen CR, Duursma RA, López R, Medlyn BE (2018). Triggers of tree mortality under drought. Nature, 558, 531-539.

Choat B, Jansen S, Brodribb TJ, Cochard H, Delzon S, Bhaskar R, Bucci SJ, Feild TS, Gleason SM, Hacke UG, Jacobsen AL, Lens F, Maherali H, Martínez-Vilalta J, Mayr S, Mencuccini M, Mitchell PJ, Nardini A, Pittermann J, Pratt RB, Sperry JS, Westoby M, Wright IJ, Zanne AE (2012). Global convergence in the vulnerability of forests to drought. Nature, 491, 752-755.

Choat B, Medek DE, Stuart SA, Pasquet-Kok J, Egerton JJG, Salari H, Sack L, Ball MC (2011). Xylem traits mediate a trade-off between resistance to freeze-thaw-induced embolism and photosynthetic capacity in overwintering evergreens. New Phytologist, 191, 996-1005.

Christensen-Dalsgaard KK, Tyree MT (2013). Does freezing and dynamic flexing of frozen branches impact the cavitation resistance of Malus domestica and the Populus clone walker? Oecologia, 173, 665-674.

Christensen-Dalsgaard KK, Tyree MT (2014). Frost fatigue and spring recovery of xylem vessels in three diffuse-porous trees in situ. Plant, Cell \& Environment, 37, 1074-1085.

Climent J, Costa e Silva F, Chambel MR, Pardos M, Almeida MH (2009). Freezing injury in primary and secondary needles of Mediterranean pine species of contrasting ecological niches. Annals of Forest Science, 66, 407. DOI: 10.1051/forest/2009016.

Cochard H, Tyree MT (1990). Xylem dysfunction in Quercus: Vessel sizes, tyloses, cavitation and seasonal changes in embolism. Tree Physiology, 6, 393-407.

Davis SD, Sperry JS, Hacke UG (1999). The relationship between xylem conduit diameter and cavitation caused by freezing. American Journal of Botany, 86, 1367-1372.

de Swaef T, Hanssens J, Cornelis A, Steppe K (2013). Non-destructive estimation of root pressure using sap flow, stem diameter measurements and mechanistic modelling. Annals of Botany, 111, 271-282.

Domec JC (2011). Let's not forget the critical role of surface tension in xylem water relations. Tree Physiology, 31, 359-360.

Feng F, Ding F, Tyree MT (2015). Investigations concerning cavitation and frost fatigue in clonal $84 \mathrm{~K}$ poplar using high-resolution cavitron measurements. Plant Physiology, 168, 144-155.

Fernández-Pérez L, Villar-Salvador P, Martínez-Villata J, Toca A, Zavala MA (2018). Distribution of pines in the Iberian Peninsula agrees with species differences in foliage frost 
tolerance, not with vulnerability to freezing-induced xylem embolism. Tree Physiology, 38, 507-516.

Feild TS, Brodribb T (2001). Stem water transport and freeze-thaw xylem embolism in conifers and angiosperms in a Tasmanian treeline heath. Oecologia, 127, 314-320.

Gleason SM, Blackman CJ, Gleason ST, McCulloh KA, Ocheltree TW, Westoby M (2018). Vessel scaling in evergreen angiosperm leaves conforms with Murray's law and area-filling assumptions: Implications for plant size, leaf size and cold tolerance. New Phytologist, 218, 1360-1370.

Gleason SM, Westoby M, Jansen S, Choat B, Hacke UG, Pratt RB, Bhaskar R, Brodribb TJ, Bucci SJ, Cao KF, Cochard H, Delzon S, Domec JC, Fan ZX, Feild TS, Jacobsen AL, Johnson DM, Lens F, Maherali H, Martínez-Vilalta J, Mayr S, McCulloh KA, Mencuccini M, Mitchell PJ, Morris H, Nardini A, Pittermann J, Plavcová L, Schreiber SG, Sperry JS, Wright IJ, Zanne AE (2016). Weak tradeoff between xylem safety and xylem-specific hydraulic efficiency across the world's woody plant species. New Phytologist, 209, 123-136.

Granda E, Scoffoni C, Rubio-Casal AE, Sack L, Valladares F (2014). Leaf and stem physiological responses to summer and winter extremes of woody species across temperate ecosystems. Oikos, 123, 1281-1290.

Hacke UG, Lachenbruch B, Pittermann J, Mayr S, Domec JC, Schulte PJ (2015). The hydraulic architecture of conifers. In: Hacke U ed. Functional and Ecological Xylem Anatomy. Springer, Cham, Switzerland. 39-75.

Hacke UG, Sperry JS (2001). Functional and ecological xylem anatomy. Perspectives in Plant Ecology, Evolution and Systematics, 4, 97-115.

Hacke UG, Spicer R, Schreiber SG, Plavcová L (2017). An ecophysiological and developmental perspective on variation in vessel diameter. Plant, Cell \& Environment, 40, 831-845.

Hajek P, Leuschner C, Hertel D, Delzon S, Schuldt B (2014). Trade-offs between xylem hydraulic properties, wood anatomy and yield in Populus. Tree Physiology, 34, 744-756.

Hao GY, Hoffmann WA, Scholz FG, Bucci SJ, Meinzer FC, Franco AC, Cao KF, Goldstein G (2008). Stem and leaf hydraulics of congeneric tree species from adjacent tropical savanna and forest ecosystems. Oecologia, 155, 405-415.

Hao GY, Wheeler JK, Holbrook NM, Goldstein G (2013). Investigating xylem embolism formation, refilling and water storage in tree trunks using frequency domain reflectometry. Journal of Experimental Botany, 64, 2321-2332.

Hillabrand RM, Hacke UG, Lieffers VJ (2016). Drought-induced xylem pit membrane damage in aspen and balsam poplar. Plant, Cell \& Environment, 39, 2210-2220.

Hoermiller II, Ruschhaupt M, Heyer AG (2018). Mechanisms of frost resistance in Arabidopsis thaliana. Planta, 248, 827-835.

Hoffman L, DaCosta M, Ebdon JS, Zhao JZ (2012). Effects of drought preconditioning on freezing tolerance of perennial ryegrass. Environmental and Experimental Botany, 79, 11-20.

Hoffmann WA, Marchin RM, Abit P, Lau OL (2011). Hydraulic failure and tree dieback are associated with high wood density in a temperate forest under extreme drought. Global Change Biology, 17, 2731-2742.

Hölttä T, Dominguez Carrasco MDR, Salmon Y, Aalto J, Vanhatalo A, Bäck J, Lintunen A (2018). Water relations in silver birch during springtime: How is sap pressurised? Plant Biology, 20, 834-847.

Jiménez-Castillo M, Lusk CH (2013). Vascular performance of woody plants in a temperate rain forest: Lianas suffer higher levels of freeze-thaw embolism than associated trees. Functional Ecology, 27, 403-412.

Jin Y, Wang CK, Zhou ZH, Li ZM (2016). Co-ordinated performance of leaf hydraulics and economics in 10 Chinese temperate tree species. Functional Plant Biology, 43, 1082-1090.

Jin Y, Wang CK (2016). Leaf hydraulic traits and their trade-offs for nine Chinese temperate tree species with different wood properties. Chinese Journal of Plant Ecology, 40, 702-710. [金鹰，王传宽 (2016). 九种不同材性 的温带树种叶水力性状及其权衡关系. 植物生态学报, 40, 702-710.]

Jin Y, Wang CK, Zhou ZH (2016). Mechanisms of xylem embolism repair in woody plants: Research progress and questions. Chinese Journal of Plant Ecology, 40, 834-846. [金鹰，王传宽，周正虎 (2016). 木本植物木质部栓塞 修复机制: 研究进展与问题。植物生态学报, 40 , 834-846.]

Johnson DM, McCulloh KA, Woodruff DR, Meinzer FC (2012). Hydraulic safety margins and embolism reversal in stems and leaves: Why are conifers and angiosperms so different? Plant Science, 195, 48-53.

Kong RS, Henry HAL (2016). Prior exposure to freezing stress enhances the survival and recovery of Poa pratensis exposed to severe drought. American Journal of Botany, 103, 1890-1896.

Kreyling J, Schmid S, Aas G (2015). Cold tolerance of tree species is related to the climate of their native ranges. Journal of Biogeography, 42, 156-166.

Kreyling J, Wiesenberg GLB, Thiel D, Wohlfart C, Huber G, Walter J, Jentsch A, Konnert M, Beierkuhnlein C (2012). Cold hardiness of Pinus nigra Arnold as influenced by geographic origin, warming, and extreme summer drought. Environmental and Experimental Botany, 78, 99-108.

Lemoine D, Granier A, Cochard H (1999). Mechanism of freeze-induced embolism in Fagus sylvatica L. Trees, 13,

www.plant-ecology.com 
206-210.

Lens F, Sperry JS, Christman MA, Choat B, Rabaey D, Jansen S (2011). Testing hypotheses that link wood anatomy to cavitation resistance and hydraulic conductivity in the genus Acer. New Phytologist, 190, 709-723.

Lens F, Tixier A, Cochard H, Sperry JS, Jansen S, Herbette S (2013). Embolism resistance as a key mechanism to understand adaptive plant strategies. Current Opinion in Plant Biology, 16, 287-292.

Lindfors L, Hölttä T, Lintunen A, Porcar-Castell A, Nikinmaa E, Juurola E (2015). Dynamics of leaf gas exchange, chlorophyll fluorescence and stem diameter changes during freezing and thawing of Scots pine seedlings. Tree Physiology, 35, 1314-1324.

Lintunen A, Hölttä T, Kulmala M (2013). Anatomical regulation of ice nucleation and cavitation helps trees to survive freezing and drought stress. Scientific Reports, 3, 2031. DOI: $10.1038 /$ srep02031.

Lintunen A, Lindfors L, Kolari P, Juurola E, Nikinmaa E, Hölttä T (2014). Bursts of $\mathrm{CO}_{2}$ released during freezing offer a new perspective on avoidance of winter embolism in trees. Annals of Botany, 114, 1711-1718.

Lintunen A, Lindfors L, Nikinmaa E, Hölttä T (2016). Xylem diameter changes during osmotic stress, desiccation and freezing in Pinus sylvestris and Populus tremula. Tree Physiology, 37, 491-500.

Lintunen A, Mayr S, Salmon Y, Cochard H, Hölttä T (2018). Drivers of apoplastic freezing in gymnosperm and angiosperm branches. Ecology and Evolution, 8, 333-343.

Losso A, Nardini A, Dämon B, Mayr S (2018). Xylem sap chemistry: Seasonal changes in timberline conifers Pinus cembra, Picea abies, and Larix decidua. Biologia Plantarum, 62, 157-165.

Martínez-Vilalta J, Sala AN, Asensio D, Galiano L, Hoch G, Palacio S, Piper FI, Lloret F (2016). Dynamics of non-structural carbohydrates in terrestrial plants: A global synthesis. Ecological Monographs, 86, 495-516.

Mayr S, Améglio T (2016). Freezing stress in tree xylem. Progress in Botany, 77, 381-414.

Mayr S, Cochard H, Améglio T, Kikuta SB (2007). Embolism formation during freezing in the wood of Picea abies. Plant Physiology, 143, 60-67.

Mayr S, Hacke U, Schmid P, Schwienbacher F, Gruber A (2006). Frost drought in conifers at the alpine timberline: Xylem dysfunction and adaptations. Ecology, 87, 3175-3185.

Mayr S, Schmid P, Laur J, Rosner S, Charra-Vaskou K, Dämon B, Hacke UG (2014). Uptake of water via branches helps timberline conifers refill embolized xylem in late winter. Plant Physiology, 164, 1731-1740.

Mayr S, Schwienbacher F, Bauer H (2003). Winter at the alpine timberline. Why does embolism occur in Norway spruce but not in stone pine? Plant Physiology, 131, 780-792.
Mayr S, Sperry JS (2010). Freeze-thaw-induced embolism in Pinus contorta: Centrifuge experiments validate the 'thawexpansion hypothesis' but conflict with ultrasonic emission data. New Phytologist, 185, 1016-1024.

Mayr S, Wolfschwenger M, Bauer H (2002). Winter-drought induced embolism in Norway spruce (Picea abies) at the Alpine timberline. Physiologia Plantarum, 115, 74-80.

McCully ME, Canny MJ, Huang CX (2004). The management of extracellular ice by petioles of frost-resistant herbaceous plants. Annals of Botany, 94, 665-674.

McDowell NG (2011). Mechanisms linking drought, hydraulic, carbon metabolism, and vegetation mortality. Plant Physiology, 155, 1051-1059.

McDowell N, Pockman WT, Allen CD, Breshears DD, Cobb N, Kolb T, Plaut J, Sperry J, West A, Williams DG, Yepez EA (2008). Mechanisms of plant survival and mortality during drought: Why do some plants survive while others succumb to drought? New Phytologist, 178, 719-739.

Medeiros JS, Danielson SC (2018). Renewed interest in whole-plant physiology sheds light on the complexity of plant stress response architecture. Tree Physiology, 38, 503-506.

Medeiros JS, Pockman WT (2011). Drought increases freezing tolerance of both leaves and xylem of Larrea tridentate. Plant, Cell \& Environments, 34, 43-51.

Nardini A, Lo Gullo MA, Salleo S (2011). Refilling embolized xylem conduits: Is it a matter of phloem unloading? Plant Science, 180, 604-611.

Nardini A, Luglio J (2014). Leaf hydraulic capacity and drought vulnerability: Possible trade-offs and correlations with climate across three major biomes. Functional Ecology, 28, 810-818.

Nardini A, Pedà G, La Rocca N (2012). Trade-offs between leaf hydraulic capacity and drought vulnerability: Morphoanatomical bases, carbon costs and ecological consequences. New Phytologist, 196, 788-798.

Niu CY, Meinzer FC, Hao GY (2017). Divergence in strategies for coping with winter embolism among co-occurring temperate tree species: The role of positive xylem pressure, wood type and tree stature. Functional Ecology, 31, 1550-1560.

Pittermann J, Sperry J (2003). Tracheid diameter is the key trait determining the extent of freezing-induced embolism in conifers. Tree Physiology, 23, 907-914.

Pittermann J, Sperry JS (2006). Analysis of freeze-thaw embolism in conifers. The interaction between cavitation pressure and tracheid size. Plant Physiology, 140, 374-382.

Pivovaroff AL, Sack L, Santiago LS (2014). Coordination of stem and leaf hydraulic conductance in southern California shrubs: A test of the hydraulic segmentation hypothesis. New Phytologist, 203, 842-850.

Ployet R, Soler M, Carocha V, Ladouce N, Alves A, Rodrigues 
J, Harvengt L, Marque C, Teulières C, Grima-Pettenati J, Mounet F (2018). Long cold exposure induces transcriptional and biochemical remodelling of xylem secondary cell wall in Eucalyptus. Tree Physiology, 38, 409-422.

Prendin AL, Mayr S, Beikircher B, von Arx G, Petit G (2018). Xylem anatomical adjustments prioritize hydraulic efficiency over safety as Norway spruce trees grow taller. Tree Physiology, 38, 1088-1097.

Preston JC, Sandve SR (2013). Adaptation to seasonality and the winter freeze. Frontiers in Plant Science, 4, 167. DOI: 10.3389/fpls.2013.00167.

Rasmussen DH, MacKenzie AP (1972). Effect of solute on ice-solution interfacial free energy; calculation from measured homogeneous nucleation temperatures. In: Jellinek HHG ed. Water Structure at the Water-polymer Interface. Springer, Boston. 126-145.

Reich PB (2014). The world-wide "fast-slow" plant economics spectrum: A traits manifesto. Journal of Ecology, 102, 275-301.

Reich PB, Sendall KM, Stefanski A, Wei XR, Rich RL, Montgomery RA (2016). Boreal and temperate trees show strong acclimation of respiration to warming. Nature, 531, 633-636.

Reyes-Díaz M, Ulloa N, Zúñiga-Feest A, Gutiérrez A, Gidekel M, Alberdi M, Corcuera LJ, Bravo LA (2006). Arabidopsis thaliana avoids freezing by supercooling. Journal of Experimental Botany, 57, 3687-3696.

Rorato DG, Araujo MM, Tabaldi LA, Turchetto F, Griebeler AM, Berghetti ÁLP, Barbosa FM (2018). Tolerance and resilience of forest species to frost in restoration planting in southern Brazil. Restoration Ecology, 26, 537-542.

Salleo S, Lo Gullo MA, Trifilò P, Nardini A (2004). New evidence for a role of vessel-associated cells and phloem in the rapid xylem refilling of cavitated stems of Laurus nobilis L. Plant, Cell \& Environment, 27, 1065-1076.

Santiago LS, De Guzman ME, Baraloto C, Vogenberg JE, Brodie M, Hérault B, Fortunel C, Bonal D (2018). Coordination and trade-offs among hydraulic safety, efficiency and drought avoidance traits in Amazonian rainforest canopy tree species. New Phytologist, 218, 1015-1024.

Schreiber SG, Hamann A, Hacke UG, Thomas BR (2013). Sixteen years of winter stress: An assessment of cold hardiness, growth performance and survival of hybrid poplar clones at a boreal planting site. Plant, Cell \& Environment, 36, 419-428.

Sevanto S, Suni T, Pumpanen J, Grönholm T, Kolari P, Nikinmaa E, Hari P, Vesala T (2006). Wintertime photosynthesis and water uptake in a boreal forest. Tree Physiology, 26, 749-757.

Sierra-Almeida A, Reyes-Bahamonde C, Cavieres LA (2016). Drought increases the freezing resistance of high-elevation plants of the Central Chilean Andes. Oecologia, 181,
$1011-1023$

Sperling O, Secchi F, Godfrey J, Zwieniecki MA (2017). Acclimation of Pistacia integerrima trees to frost in semi-arid environments depends on autumn's drought. Planta, 245, 671-679.

Sperry JS, Hacke UG, Pittermann J (2006). Size and function in conifer tracheids and angiosperm vessels. American Journal of Botany, 93, 1490-1500.

Sperry JS, Love DM (2015). What plant hydraulics can tell us about responses to climate-change droughts. New Phytologist, 207, 14-27.

Sperry JS, Sullivan JEM (1992). Xylem embolism in response to freeze-thaw cycles and water stress in ring-porous, diffuse-porous, and conifer species. Plant Physiology, 100, 605-613.

Stiller V, Sperry JS (2002). Cavitation fatigue and its reversal in sunflower (Helianthus annuus L.). Journal of Experimental Botany, 53, 1155-1161.

Taneda H, Tateno M (2005). Hydraulic conductivity, photosynthesis and leaf water balance in six evergreen woody species from fall to winter. Tree Physiology, 25, 299-306.

Toca A, Oliet JA, Villar-Salvador P, Maroto J, Jacobs DF (2018). Species ecology determines the role of nitrogen nutrition in the frost tolerance of pine seedlings. Tree Physiology, 38, 96-108.

Tyree MT, Ewers FW (1991). The hydraulic architecture of trees and other woody plants. New Phytologist, 119, 345-360.

Venturas MD, Sperry JS, Hacke UG (2017). Plant xylem hydraulics: What we understand, current research, and future challenges. Journal of Integrative Plant Biology, 59, 356-389.

Verhoeven A, García-Plazaola JI, Fernández-Marín B (2018). Shared mechanisms of photoprotection in photosynthetic organisms tolerant to desiccation or to low temperature. Environmental and Experimental Botany, 154, 66-79.

Vitasse Y, Lenz A, Körner C (2014). The interaction between freezing tolerance and phenology in temperate deciduous trees. Frontiers in Plant Science, 5, 541. DOI: 10.3389/fpls.2014.00541.

Walter J, Jentsch A, Beierkuhnlein C, Kreyling J (2013). Ecological stress memory and cross stress tolerance in plants in the face of climate extremes. Environmental and $E x$ perimental Botany, 94, 3-8.

Wang AY, Han SJ, Zhang JH, Wang M, Yin XH, Fang LD, Yang D, Hao GY (2018). The interaction between nonstructural carbohydrate reserves and xylem hydraulics in Korean pine trees across an altitudinal gradient. Tree Physiology, 38, 1792-1804.

Westhoff M, Schneider H, Zimmermann D, Mimietz S, Stinzing A, Wegner LH, Kaiser W, Krohne G, Shirley S, Jakob P, Bamberg E, Bentrup FW, Zimmermann U

www.plant-ecology.com 
(2008). The mechanisms of refilling of xylem conduits and bleeding of tall birch during spring. Plant Biology, 10, 604-623.

Willick IR, Gusta LV, Fowler DB, Tanino KK (2019). Ice segregation in the crown of winter cereals: Evidence for extraorgan and extratissue freezing. Plant, Cell \& Environment, 42, 701-716.

Willick IR, Takahashi D, Fowler DB, Uemura M, Tanino KK (2018). Tissue-specific changes in apoplastic proteins and cell wall structure during cold acclimation of winter wheat crowns. Journal of Experimental Botany, 69, 1221-1234.

Willson CJ, Jackson RB (2006). Xylem cavitation caused by drought and freezing stress in four co-occurring Juniperus species. Physiologia Plantarum, 127, 374-382.

Yin XH, Hao GY (2018). Divergence between ring- and diffuseporous wood types in broadleaf trees of Changbai Mountains results in substantial differences in hydraulic traits. Chinese Journal of Applied Ecology, 29, 352-360. [殷笑 寒, 郝广友 (2018). 长白山阔叶树种木质部环孔和散孔 结构特征的分化导致其水力学性状的显著差异. 应用 生态学报, 29, 352-360.]

Yin XH, Sterck F, Hao GY (2018). Divergent hydraulic strategies to cope with freezing in co-occurring temperate tree species with special reference to root and stem pressure generation. New Phytologist, 219, 530-541.

Zanne AE, Tank DC, Cornwell WK, Eastman JM, Smith SA, FitzJohn RG, McGlinn DJ, O’Meara BC, Moles AT, Reich
PB, Royer DL, Soltis DE, Stevens PF, Westoby M, Wright IJ, Aarssen L, Bertin RI, Calaminus A, Govaerts R, Hemmings F, Leishman MR, Oleksyn J, Soltis PS, Swenson NG, Warman L, Beaulieu JM (2014). Three keys to the radiation of angiosperms into freezing environments. $\mathrm{Na}$ ture, 506, 89-92.

Zhang HY, Wang CK, Wang XC (2013). Comparison of concentrations of non-structural carbohydrates between new twigs and old branches for 12 temperate species. Acta Ecologica Sinica, 33, 5675-5685. [张海燕, 王传宽, 王兴 昌 (2013). 温带12个树种新老树枝非结构性碳水化合 物浓度比较. 生态学报, 33, 5675-5685.]

Zhang W, Feng F, Tyree MT (2018). Seasonality of cavitation and frost fatigue in Acer mono Maxim. Plant, Cell \& Environment, 41, 1278-1286.

Zhang YJ, Bucci SJ, Arias NS, Scholz FG, Hao GY, Cao KF, Goldstein G (2016). Freezing resistance in Patagonian woody shrubs: The role of cell wall elasticity and stem vessel size. Tree Physiology, 36, 1007-1018.

Zhu SD, Li RH, He PC, Siddiq Z, Cao KF, Ye Q (2019). Large branch and leaf hydraulic safety margins in subtropical evergreen broadleaved forest. Tree Physiology, 39, 1405-1415.

Zimmermann MH (1983). Xylem Structure and the Ascent of Sap. Spring, Berlin.

责任编委: 杜 盛 责任编辑: 李 敏 实习编辑: 赵 航 